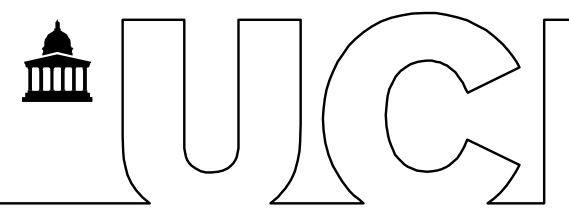

Gainforth, HL; West, R; Michie, S; (2015) Assessing Connections Between Behavior Change Theories Using Network Analysis. Annals of Behavioral Medicine, 49 (5) pp. 754-761.

10.1007/s12160-015-9710-7. Downloaded from UCL Discovery: http://discovery.ucl.ac.uk/1468913

\title{
ARTICLE
}

\section{Assessing connections between behavior change theories using network analysis}

\author{
Gainforth, Heather L. '; West, Robert ${ }^{2}$; Michie, Susan ${ }^{1}$ \\ ${ }^{1}$ Research Department of Clinical, Educational and Health Psychology, Centre for \\ Outcomes Research and Effectiveness (CORE), University College London \\ ${ }^{2}$ Department of Epidemiology and Public Health, University College London
}

\begin{abstract}
Background: A cross-disciplinary scoping review of behavior change theories identified 83, with many similarities and overlapping constructs. Investigating the derivation of these theories may provide further understanding of their contribution and intended application.

Purpose: To develop and apply a method to describe the explicit derivation of theories of behavior change.

Methods: A network analysis of the explicit 'contributing to' relations between the 83 theories was conducted. Identification of relations involved textual analysis of primary theory sources. Findings: 122 connections between the theories were identified amounting to $1.8 \%$ of the number possible. On average, theories contributed to one or two theories (Mean = $1.47 \pm 3.69$ contributions) and were informed by one or two theories (Mean $=1.47 \pm 1.61$ contributing theories).
\end{abstract}

Discussion: Most behavior change theories appear to be explicitly informed by few prior theories. If confirmed, this suggests a considerable dislocation between generations of theories and would be expected to undermine scientific progress.

Keywords: network analysis, behavior change, theory, intervention development

\section{Introduction}

Interventions and policies that aim to effect even small changes in health behaviors can lead to substantial improvements to public health (1-6). Research evaluating health behavior change interventions targeting a variety of behaviors at the individual, community and population levels indicates that success of behavior change interventions is varied with many examples of both successful and ineffective interventions (5-13).

Systematic application of appropriate theories of behavior and behavior change (hereinafter referred to as theories of behavior change) may support the development of effective behavior change interventions in several ways (14). By theory, we mean 'a set of concepts and/or statements with specification of how phenomena relate to each other' (a consensus definition of a multidisciplinary expert group of psychologists, sociologists, anthropologists and economists) (15). Theories of behavior change summarise the cumulative knowledge of mechanisms and moderators of change across different populations, behaviors and contexts and thus provide an organising description of a system that accounts for what is known, and 
explains and predicts phenomena ${ }^{15}$. By linking theoretical constructs and mechanisms of action to behavior change techniques, intervention developers can use theoretical understanding of behaviors in their contexts to select, refine and tailor behavior change techniques and intervention evaluators can investigate mechanisms of action that brought about intervention effects (14). Some reviews have found a positive association between the use of theory in intervention development and effectiveness of interventions (10, 16-20), while other reviews have found mixed, null or even negative results (21-23). These mixed findings may be explained by the variable manner in which theories are selected and used to develop interventions $(24,25)$.

Given theory's potential value for intervention development, there is increasing recognition that the development and evaluation of behavior change interventions should draw on theories of behavior change $(16,17,26-28)$. For example, the UK's Medical Research Council guidance for complex interventions recommends that those developing and evaluating interventions should identify relevant theories to understand the process of change and apply them to intervention development and evaluation (26-28). Despite the potential advantages of using theory to develop behavior change interventions, they are often designed without reference to theory. Reviews of empirical research articles have found that the minority of interventions use theory to inform their development or evaluation, in areas of preventive health behaviors where $35.7 \%$ were found to refer to theory (29) and of implementation strategies where only $23 \%$ of studies explicitly used theory (30).

There are several reasons why researchers do not use theory. For example, they may not consider it helpful, or they may lack the skills to select or apply theory, especially given the lack of guidance in this area. The selection of theory is especially challenging given the sheer numbers of overlapping theories and constructs in behavior change. For example, a consensus method generated 33 theories and 128 explanatory constructs deemed as relevant to studying the implementation of evidence based practice(31). Similarly, a recent scoping review of behavior change theories across social and behavioral sciences identified 83 theories with many similarities and numerous overlapping constructs (15). Despite this, the published research in behavior change is dominated by a handful of theories. For example, a review of 193 empirical health behavior research articles published from 2000 to 2005 found that $35.7 \%$ mentioned theory with the most-often-used theories being the Transtheoretical Model (27.5\%), Social Cognitive Model (27.5\%), and the Health Belief Model (20\%) (29). It is likely, therefore, that there is untapped potential in the many theories that are rarely used $(24,29)$.

One method for guiding theory selection is to use a framework that has been developed through expert-consensus and integrates theories and theoretical constructs into general domains that can be used to assess problems and develop interventions. Two such frameworks are the COM-B model and the Theoretical Domains Framework (TDF) (31-34). The COM-B model of behavior seeks to provide a simple but comprehensive framework within which other theories can be considered. The model conceptualizes behavior as part of a mutually influencing system in which capability, opportunity and motivation interact to change behavior $(14,35)$. Developed by psychologists and implementation researchers, the TDF further elaborates on the three sources of behavior in the COM-B by outlining 14 theoretical domains that should be considered when developing interventions $(32,36)$. While these methods provide a theoretical basis for intervention development and point to the types of theory that may be relevant, the TDF and COM-B do not provide guidance for selecting specific theories. An alternative approach to simplifying the plethora of overlapping theories is to further our understanding of how theories relate to each other in terms of derivation and overlapping constructs.

One method for investigating the derivation and relationships among theories, including links, patterns and gaps, is network analysis. This research approach aims to describe, 
explore and understand the structural and relational aspects of a group or network (37). Applying a network analysis could be used to investigate the derivation of behavior change theories by describing how or if reported connections exist among theories, identifying theories that contributed to the development of other theories or are based on a number of other theories, and by identifying a group of core theories that are central in the network. In turn, this data may be used by theory developer(s) to consider, test and integrate a broader set of theories.

To conduct a traditional network analysis, network data are collected by asking actors in a network to name individuals in their network. To apply this approach to theory, a method for reliably extracting contributing theory names from articles describing the development of each theory is necessary. The aims of this study were twofold: first, to develop a method for extracting the names of theories that lead to the derivation of a theory as stated by the theory developer(s); secondly, to apply the method to conduct a network analysis describing the derivation of 83 theories of behavior change identified in the full report of the scoping review (the book 'ABC of Behavior Change Theories'(14)).

\section{Method}

Design

A cross-sectional, whole-network design (i.e. network data collected for each theory in the network) was used to investigate the explicit 'contributing to' relations between theories of behavior and behavior change $(n=83)$.

\section{Theories}

The 83 theories were identified in a scoping review of theories of behavior and behavior change (see Supplementary File 1)(14, 15). A summary of each theory as originally described by the theory developer(s) (i.e. the primary theory source) was used as the data source to extract the names of contributing theories $(14,15)$. When unable to identify the primary theory source, an alternative publication that included a comprehensive description of the theory authored by the theory developer(s) was used. If a theory was refined or expanded by its original developer(s) using the same theory name, data were also extracted from publications reporting these refinements or expansions.

\section{Development of the Data Extraction Method}

Data were extracted from the primary theories sources by at least one pair of individuals. The data extraction protocol was developed in four rounds, with two pairs of data extractors testing out the data extraction protocol on two randomly selected theories in the first round, eight theories in the second round and a different eight theories in the third round. Between each round, the two pairs of data extractors discussed any uncertainties or discrepancies with each other and the lead researcher $(\mathrm{HG})$. The protocol was revised between each round. Given substantial inter-coder agreement at round 3, the revised data extraction protocol was used in round 4 . To reduce coder burden a third coding pair was added in round 4 and each of the three pairs extracted data from 21 or 22 theories.

Data extraction protocol

The initial data extraction protocol was developed by the lead researcher ( $\mathrm{HG}$ ) and the principal investigator (SM). Pairs of researchers read randomly selected primary theory sources from the full report of the scoping review (the book 'ABC of Behavior Change Theories' (14)), followed by the original published article from which the summary was drawn. They extracted names of theories identified as informing the development of each theory together with the rationale for each decision to include or exclude a potential contributing theory (data extraction form is in Supplementary File 2). To be deemed a contributing theory, the theory developer(s) must have indicated in the theory source that the theory contributed to the development of one or more constructs within their theory. The lead 
researcher reviewed data extractors' decisions, discussed any discrepancies and finalised the contributing theory list.

The initial data extraction protocol was revised to account for ambiguities in theory descriptions. Data extractors reported difficulty identifying 1) the difference between a parent theory (i.e. contributing theory) and a peer theory (i.e. a similar or contrasting theory that did not contribute to development); and 2) theories not identified in the scoping review that informed the development of the theory. To address these ambiguities within primary theory sources, data were extracted only when the theory developer(s) clearly and explicitly stated that a specific theory contributed to the development of their theory or a construct within their theory. Mere mention of a theory in reviewing theoretical literature or comparing theories was not included as a contributing theory.

Analysis

Reliability of Method

Agreement between pairs of data extractors was assessed following rounds 2, 3 and 4 using the adjusted Kappa statistic (i.e. PABAK)(38). While we also calculated percentage agreement and Cohen's Kappa statistic(39), PABAK was used because of its adjustment for 1) data extractors sharing bias in the use of categories and 2) high prevalence of negative agreement (i.e. when both data extractors agree on non-contributing theories). Inter-coder agreement values of $0.60-0.79$ indicate 'substantial' reliability and those above 0.80 are 'outstanding' (40). Main themes from challenges reported by data extractors were identified and illustrative comments for each theme were selected.

Network Analysis

The resulting network analysis was performed using UCINET v6 (41) and NETDRAW (42) software. Network measures used to describe the derivation of behavior change theories included network density, degree centrality (i.e. out-degree and in-degree), and assessment of the core-periphery structure. The definition and relevance of each of these measures is described below.

\section{Network Density}

This measure refers to the number of connections in the network as a proportion of all possible ties in the network $(43,44)$. Density is calculated by dividing the number of connections in the network by the total number of links possible within the network (44). In the context of this analysis, the network density measure was used to determine how or if potential connections exist between theories in the network. To understand how the range of theory publication dates (i.e. 1922 - 2014) may have affected this measure, network density was examined across three date ranges: 1) $1922-1985$ (27 theories; i.e. 1/3 of the whole network); 2) 1922 - 1999 (54 theories; i.e. 2/3 of the whole network); and 3) 1922 2014 (83 theories, i.e. the whole network).

Degree centrality

Centrality is a measure of the extent to which a theory occupies a central position (44). Degree centrality is a directional measure of centrality that refers to the number of links to and from a theory and is calculated without reference to the overall structure of the network (44). As this measure is directional, one can measure both the number of outgoing ties from a theory (i.e. out-degree centrality) and the number of incoming ties to a theory (i.e. indegree centrality). In the context of this analysis, out-degree centrality was used to identify theories that contributed to the development of other theories and in-degree centrality was used to identify theories that are based on other theories.

To account for range of theory publication dates, a percent out- and in-degree score was calculated. For each theory, the total number of theories that the theory could have contributed to (i.e. out-degree centrality) and been based on (i.e. in-degree centrality) when 
the theory was published was used as the denominator and the raw in- and out-degree centrality scores were used as the numerator.

Core-periphery structure

This measure assesses whether the network exhibits a core-periphery structure. A coreperiphery is a network structure that consists of a group of theories that are densely connected to one another (i.e. the core) and a separate group that are loosely connected to the core (i.e. the periphery) (44). In the context of this analysis, this measure was used to extend the notion of centrality to groups of theories within the network.

\section{Results}

Method Development

Reliability

Across all coding rounds, average inter-coder agreement was substantial (Kappa $=0.68 \pm$ 0.41 ; PABAK $=0.99 \pm 0.03$ ). The initial data extraction protocol revealed moderate agreement levels, but reliability improved following refinement of the protocol (Kappa $=0.70 \pm 0.40$; PABAK $=\quad 0.99 \pm 0.02$; see last 4 columns of Supplementary file 1 and Table 1).

Challenges reported by data extractors

Data extractors reported two main challenges. The first related to the ambiguity with which theory developer(s) described the development and origins of their theory. Authors frequently referred to a broad scope of literature or theory rather than outlining specific names of theories or constructs that contributed to the development of their theory. Illustrative comments include:

'(Theory Developers) seems to draw influence from many theories, but only explicitly cites Fishbein' (Coder 1, Social Ecological Model for Behavior Change).

'Discusses the scientific background leading to his research, but as there's a wide base of evidence for his research, it's difficult to pinpoint specific theories that contributed' (Coder 1, Classical Conditioning).

'Lists theories it is inspired by in bulk in an early sentence but not in detail' (Coder 6, AIDS Risk Reduction Model).

The second main challenge related to ambiguity in the way in which theory developer(s) reported contributing theories (parent theory) as opposed to similar or contrasting theories that did not contribute to development (peer theory). Data extractors found it difficult to discern whether authors were referring to parent or peer theories as theory developer(s) did not explicitly state which theories contributed to the development of their theory. Illustrative comments include:

'Bandura \& Ajzen both cited in references, but author highlights a construct from the theory and then critiques it, he doesn't build on this critique, rather highlights why his model is different' (Coder 5, The Reflective Impulsive Model).

'It was difficult to identify contributing theories because the author does not specify which theory is integrated to his model.' (Coder 3, Integrated Theory of Drinking Behavior)

Network Analysis

Density

In total, 122 connections between the 83 theories (published between 1922 - 2014) were identified amounting to only $1.8 \%$ of the number possible (see Figure 1). Density decreased minimally overtime. From 1922 - 1985, 21 connections between the 27 theories published 
were identified amounting to $3.0 \%$ of the number possible. From $1922-1999,66$ connections between the 53 theories published were identified amounting to $2.3 \%$ of the number possible.

\section{Degree Centrality}

Raw degree centrality scores indicated that on average theories contributed to one or two theories in the network (Mean $=1.47 \pm 3.69$ contributions) and were informed by one or two theories in the network (Mean $=1.47 \pm 1.61$ contributing theories); $60.0 \%$ did not contribute to any other theory in the network. Thirty-six percent of theory developers did not name any theories within the network that contributed to the development of their theory whereas $42.2 \%$ of theory developers indicated that only one or two theories in the network contributed to the development of their theory.

Percent degree centrality scores indicated that on average theories contributed to $2.5 \%$ of theories possible at date of publication (range: $0-32 \%$ ) and were informed by $6.0 \%$ of theories available at time of publication (range: $0-100 \%$ ). Percent out-degree centrality scores indcated that a minority (5\%) of theories were cited as contributing to at least $10 \%$ of theories possible at time of publication: the Health Belief Model contributed to $19 \%$ of total possible theories; Social Cognitive Theory contributed to $23 \%$ of total possible theories; Theory of Planned Behavior contributed to $30 \%$ of total possible theories; and Self-Efficacy Theory contributed to $33 \%$ of total possible theories.

Percent in-degree centrality scores indicated that 11 theory developers named more than $10 \%$ theories available at time of publication as contributing to their theory's development: Health Action Process Approach (12.2\%); Integrated Theory of Drinking Behavior (13.0\%); Theory of Triadic Influence (13.0\%); General Theory of Deviant Behavior (14.3\%); Integrative Model of Factors Influencing Smoking and Health Attitude and Behavior Change (15.8\%); Goal Setting Theory (16.7\%); Integrative Model of Health Attitude and Behavior Change (18.8\%); Health Belief Model (25.0\%); Protection Motivation Theory (33.3\%); Social Learning Theory (50.0\%); and Operant Learning Theory (100.0\%). Supplementary File 3 provides raw and percent degree centrality scores for all 83 theories in the network.

\section{Network structure}

The pattern of densities within the network was shown to be a core-periphery structure, meaning that the structure consisted of a group of densely connected central (i.e. the core) and a separate group of loosely connected theories (i.e. the periphery) (Test Fitness $=.25)(44)$. The density of ties among the core theories was $11 \%$, among periphery theories was $0.7 \%$, from the core to the periphery $2 \%$, and from periphery to core $0.7 \%$. In total, 21 theories (25\% of the sample) were identified as being part of the core: AIDS Risk Reduction Model; Behavioral-Ecological Model of Adolescent AIDS Prevention; Change Theory; Ecological Model for Preventing Type 2 Diabetes in Youth; Health Action Process Approach; Health Belief Model; I-Change Model; Information-Motivation-Behavioral Skills Model; Integrated Theoretical Model for Alcohol and Other Drug Abuse Prevention; Integrated Theory of Drinking Behavior; Integrative Model of Behavioral Prediction; Integrative Model of Health Attitude and Behavior Change; Integrative Model of Factors Influencing Smoking and Health Attitude and Behavior Change; Pressure System Model; Protection Motivation Theory; Self-Efficacy Theory; Social Cognitive Theory; Technology Acceptance Model; Temporal Self-Regulation Theory; Theory of Planned Behavior; Theory of Triadic Influence; and the Transtheoretical Model of Behavior Change.

\section{Discussion}

A method for reliably extracting names of contributing theories in theory sources and examining their derivation using network analysis has been demonstrated. The network analysis indicated that most behavior change theories appear to be explicitly informed by very few prior theories. Few theories in the network contributed to the development of other 
theories and few theories were informed by theories in the network. A core group of 21 densely connected theories in the network were identified with Social Cognitive Theory, the Health Belief Model, the Theory of Planned Behavior and Self-Efficacy Theory being the most central theories in the network. As a whole, results indicate that the reported evolution of behavior and behavior change theory may be primarily influenced by a small subset of theories. If confirmed, this suggests a considerable dislocation between generations of theories and this would be expected to undermine scientific progress.

The challenges reported by data extractors and strict inclusion criteria necessary to develop a method for extracting names of contributing theories reported by theory developer(s) highlight how poorly many of these theories were described. Since the method could only extract the names of theories that authors reported as contributing to their theory, there are likely to be wider influences that we have not been able to capture. This limitation points to the need for theorists to be explicit not only about the definitions and nature of associations between constructs but also about influences on provenance. This limitation points to the need for guidelines for consistently and accurately reporting theory development. Guidelines have been developed to improve the standards of reporting interventions in the behavioral and social sciences (i.e. CONSORT and TIDieR) $(45,46)$. As neither of the guidelines address theory development, guidelines for reporting theory development have been proposed in the ABC of Behavior Change Theories (see Supplementary File 4)(14). These guidelines should be used as a common starting point for reporting theory development.

Theories that were central to the development of other theories in the network were also those which are popular among intervention developer(s) and focused primarily on individual-level factors relating to motivation and capability rather than on social and environmental factors $(24,29)$. Since NICE's guidance on behavior change at individual, community and population level suggest that interventions that operate at several levels simultaneously are those that are most effective (47), the desirability of considering a broader range of theories is evident. However, it appears that once theories attain a certain level of prominence, there is a tendency for other developers to start with these and suggest small changes to produce a new theory (14). If behavioral science is to advance more rapidly, a broader approach that comprehensively considers the range of theories available and current supporting data is needed and more emphasis needs to be placed on improving methods of theory development.

While our data extraction protocol and resulting network analysis represent a first step to understanding the derivation of behavior and behavior change theories, several limitations of our methodology must be acknowledged. First, the validity of our network analysis is limited by the network boundary, that is, the 83 theories of behavior and behavior change identified in one scoping review(15). While examining contributing theories not identified in the systematic review was deemed too challenging for this project, this undoubtedly led to contributing theories being omitted from the network analysis. Secondly, as previously mentioned, the validity of the data extracted from a primary theory source is dependent on theory developer(s)' description of the development of their theory. Thirdly, despite double coding and substantial reliability, methods such as these are subject to human error.

Using data mining methods made possible by electronic databases and computational algorithms to extract the names of theories from primary theories sources would likely improve the comprehensiveness and accuracy of data extraction. Taking this one step forward, machine learning techniques could be combined with human expert judgment about inclusion to form a Human-Computer Expert Network (48). This method has been shown to be replicable, relatively insensitive to the size of text units and can help to avoid human error (48). 
In conclusion, our methodology and results represent a first step to documenting the links and patterns between theories of behavior and behavior change. Results provide an indication that behavior and behavior change theories may be primarily influenced by a small subset of popular theories. By creating a reliable method for extracting names from primary theory sources and identifying connections between theories, this work points to the need for accurate reporting of theory development and may begin to aid theory and intervention developer(s) to consider, test and integrate a broader range of available theories.

\section{Reference List}

1. Michie S: Designing and implementing behaviour change interventions to improve population health. Journal of Health Services Research and Policy. 2008, 13:64-69.

2. Ezzati M, Lopez AD, Rodgers $A$, et al.: Selected major risk factors and global and regional burden of disease. Lancet. 2002, 360:1347-1360.

3. Mokdad AH, Marks JS, Stroup DF, Gerberding JL: Actual causes of death in the United States, 2000. Jama-Journal of the American Medical Association. 2004, 291:12381245.

4. Solomon S, Kington R: National efforts to promote behavior-change research: views from the Office of Behavioral and Social Sciences Research. Health education research. 2002, 17:495-499.

5. National Institute for Health and Care Excellence: Behaviour change at population, community and individual levels. London: National Institute for Health and Care Excellence, 2007.

6. National Institute for Health and Care Excellence: Behaviour change: individual approaches. London: National Institute for Health and Care Excellence, 2014.

7. Michie S, West R: Behaviour change theory and evidence: a presentation to Government. Health Psychology Review. 2013, 7:1-22.

8. Nigg CR, Allegrante JP, Ory M: Theory-comparison and multiple-behavior research: common themes advancing health behavior research. Health education research. 2002, 17:670-679.

9. Abraham C, Kelly MP, West R, Michie S: The UK National Institute for Health and Clinical Excellence public health guidance on behaviour change: a brief introduction. Psychology, Health and Medicine. 2009, 14:1-8.

10. Albarracin D, Gillette JC, Earl AN, et al.: A test of major assumptions about behavior change: a comprehensive look at the effects of passive and active HIV-prevention interventions since the beginning of the epidemic. Psychological Bulletin. 2005, 131:856897.

11. Coleman T: Do financial incentives for delivering health promotion counselling work? Analysis of smoking cessation activities stimulated by the quality and outcomes framework. Bmc Public Health. 2010, 10.

12. Summerbell CD, Waters E, Edmunds LD, et al.: Interventions for preventing obesity in children. Cochrane Database of Systematic Reviews. 2005.

13. House of Lords Science and Technology Select Committee: Behaviour Change. 2nd Report for Session 2010-2012. London, 2011.

14. Michie S, West R, Campbell R, Brown J, Gainforth HL: ABC of Behaviour Change Theories. London, United Kingdom: Silverback Publishing, 2014.

15. Davis RE, Campbell R, Hildon Z, Hobbs L, Michie S: Theories of behaviour and behaviour change across the social and behavioural sciences: a scoping review. Health Psychology Review. 2014.

16. Glanz K, Bishop DB: The Role of Behavioral Science Theory in Development and Implementation of Public Health Interventions. Annual Review of Public Health, Vol 31. 2010, 31:399-418.

17. Noar SM, Benac CN, Harris MS: Does tailoring matter? Meta-analytic review of tailored print health behavior change interventions. Psychol Bull. 2007, 133:673-693.

18. Swann C, Bowe K, Kosmin M, McCormick G: Teenage pregnancy and parenthood: a review of reviews. Evidence briefing: Health Development Agency London, 2003. 
19. Albada A, Ausems MG, Bensing JM, van Dulmen S: Tailored information about cancer risk and screening: a systematic review. Patient Education and Counseling. 2009, 77:155-171.

20. Taylor N, Conner M, Lawton R: The impact of theory on the effectiveness of worksite physical activity interventions: a meta-analysis and meta-regression. Health Psychology Review. 2011, 6:33-73.

21. Roe L, Hunt $\mathrm{P}$, Bradshaw $\mathrm{H}$, Rayner $\mathrm{M}$ : Health promotion interventions to promote healthy eating in the general population: a review: Health Education Authority London, 1997.

22. Stephenson JM, Imrie J, Sutton SR: Rigorous trials of sexual behaviour interventions in STD/HIV prevention: what can we learn from them? AIDS. 2000, 14 Supp/ 3:S115-124.

23. Gardner B, Wardle J, Poston L, Croker H: Changing diet and physical activity to reduce gestational weight gain: a meta-analysis. Obesity Reviews. 2011, 12:e602-620.

24. Prestwich A, Sniehotta FF, Whittington C, et al.: Does Theory Influence the Effectiveness of Health Behavior Interventions? Meta-Analysis. Health Psychology. 2013.

25. Michie S, Prestwich A: Are interventions theory-based? Development of a theory coding scheme. Health Psychology. 2010, 29:1.

26. Craig $\mathrm{P}$, Dieppe $\mathrm{P}$, Macintyre $\mathrm{S}$, et al.: Developing and evaluating complex interventions: the new Medical Research Council guidance. BMJ. 2008, 337.

27. Campbell M, Fitzpatrick R, Haines A, et al.: Framework for design and evaluation of complex interventions to improve health. BMJ (Clinical Research Ed.). 2000, 321:694-696.

28. Campbell NC, Murray E, Darbyshire J, et al.: Designing and evaluating complex interventions to improve health care. BMJ. 2007, 334:455-459.

29. Painter JE, Borba CPC, Hynes M, Mays D, Glanz K: The use of theory in health behavior research from 2000 to 2005: A systematic review. Annals of Behavioral Medicine. 2008, 35:358-362.

30. Davies P, Walker AE, Grimshaw JM: A systematic review of the use of theory in the design of guideline dissemination and implementation strategies and interpretation of the results of rigorous evaluations. Implementation Science. 2010, 5.

31. Michie S, Johnston M, Abraham C, et al.: Making psychological theory useful for implementing evidence based practice: a consensus approach. Quality \& Safety in Health Care. 2005, 14.26-33.

32. Cane J, O'Connor D, Michie S: Validation of the theoretical domains framework for use in behaviour change and implementation research. Implementation Science. 2012, 7.

33. Michie S, Atkins L, West R: The Behaviour Change Wheel Guide to Intervention Design and Evaluation. 2014.

34. Michie S, van Stralen M, West R: The behaviour change wheel: A new method for characterising and designing behaviour change interventions. Implementation Science. 2011, 6:42.

35. Michie S, van Stralen MM, West R: The behaviour change wheel: A new method for characterising and designing behaviour change interventions. Implementation Science. 2011, 6 .

36. Michie S, Johnston M, Abraham C, et al.: Making psychological theory useful for implementing evidence based practice: a consensus approach. Quality and safety in health care. 2005, 14:26-33.

37. Luke DA, Harris JK: Network analysis in public health: history, methods, and applications. Annual review of public health. 2007, 28:69-93.

38. Byrt T, Bishop J, Carlin J: Bias, prevalence and kappa. Journal of clinical epidemiology. 1993, 46:423-429.

39. Cohen J: Weighted kappa: Nominal scale agreement provision for scaled disagreement or partial credit. Psychological Bulletin. 1968, 70:213.

40. Landis JR, Koch GG: The measurement of observer agreement for categorical data. biometrics. 1977:159-174.

41. Borgatti SP, Everett MG, Freeman LC: UCINET for Windows: Software for Social Network Analysis. Harvard, MA: Analytic Technologies, 2002. 
42. Borgatti SP: Netdraw Network Visualization. Harvard, MA: Analytic Technologies, 2002.

43. Hanneman R, Riddle M: Introduction to Social Network Methods. Riverside, CA: University of California, 2005.

44. Valente TW: Social Networks and Health: Models, Methods, and Applications. New York: Oxford University Press, 2010.

45. Hoffmann TC, Glasziou PP, Boutron I, et al.: Better reporting of interventions: template for intervention description and replication (TIDieR) checklist and guide, 2014.

46. Moher D, Schulz KF, Simera I, Altman DG: Guidance for developers of health research reporting guidelines. PLoS medicine. 2010, 7:e1000217.

47. National Institute for Health and Care Excellence: Behaviour change: the principles for effective interventions (PH6). 2007.

48. Larsen KR, Monarchi DE, Hovorka DS, Bailey CN: Analyzing unstructured text data: Using latent categorization to identify intellectual communities in information systems. Decision Support Systems. 2008, 45:884-896. 
Table 1. Inter-coder agreement statistics including percent agreement; Kappa and PABAK and the number of observations, theories and coding pairs used during each coding phase.

\begin{tabular}{|c|c|c|c|c|c|c|c|}
\hline Phase & $n$ observations & $n$ theories & $n$ coding pairs & $\begin{array}{c}\text { Mean Percent } \\
\text { Positive Agreement } \\
( \pm S D)\end{array}$ & $\begin{array}{c}\text { Mean Percent } \\
\text { Agreement }( \pm S D)\end{array}$ & Mean Kappa $( \pm S D)$ & Mean PABAK $( \pm S D)$ \\
\hline Phase 1 & 2 & 2 & 2 & - & - & - & - \\
\hline Phase 2 & 16 & 8 & 2 & $36.35 \pm 37.77$ & $98.72 \pm 1.42$ & $0.51 \pm 0.42$ & $0.97 \pm 0.03$ \\
\hline Phase 3 & 16 & 8 & 2 & $67.08 \pm 40.25$ & $99.40 \pm 1.32$ & $0.78 \pm 0.35$ & $0.99 \pm 0.03$ \\
\hline Phase 4 & 64 & 64 & 3 & $65.08 \pm 42.26$ & $99.36 \pm 1.09$ & $0.70 \pm 0.40$ & $0.99 \pm 0.02$ \\
\hline Total & 96 & 82 & - & $58.78 \pm 42.16$ & $99.26 \pm 1.20$ & $0.68 \pm .41$ & $0.99 \pm 0.03$ \\
\hline
\end{tabular}

Note. Given the purpose of Phase 1 was to refine the initial method following coder feedback, agreement statistics were not calculated. Total number of theories coded was 82 as data was not extracted from Social Action Theory (Weber, 1978) as it was the oldest theory identified (original publication in 1922). 
Figure 1. Diagram depicting the connections between the 83 theories of behavior and behavior change

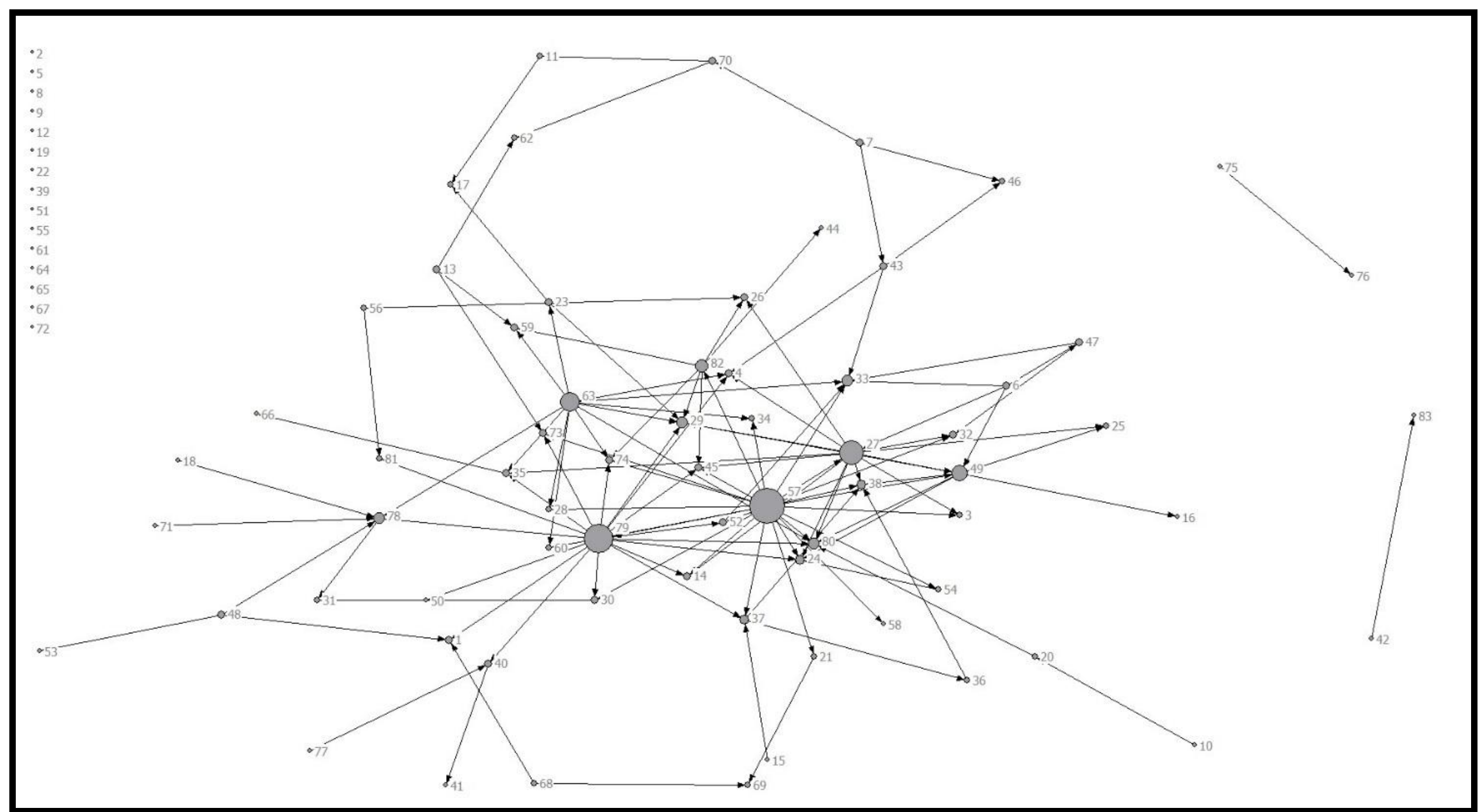

Note. Node numbers correspond to theory numbers in Supplementary File 1. Single, unconnected nodes represent theories that are not connected to any theory other theory. Node size indicates degree centrality, with larger nodes being more central in the network. 